\title{
Selective serotonin reuptake inhibitors during pregnancy and risk of persistent pulmonary hypertension in the newborn: population based cohort study from the five Nordic countries
}

\author{
(c) $\frac{(1)(8)}{\mathrm{gy}}$ MPEN ACCESS
}

Helle Kieler associate professor in obstetrics and gynaecology ${ }^{1}$, Miia Artama senior researcher ${ }^{2}$, Anders Engeland senior researcher ${ }^{34}$, Örjan Ericsson researcher ${ }^{5}$, Kari Furu professor and senior researcher $^{36}$, Mika Gissler research professor ${ }^{72}$, Rikke Beck Nielsen biostatistician ${ }^{8}$, Mette Nørgaard associate professor and senior consultant in epidemiology ${ }^{8}$, Olof Stephansson associate professor and senior consultant in obstetrics and gynaecology ${ }^{19}$, Unnur Valdimarsdottir associate professor ${ }^{10}$, Helga Zoega postdoctoral fellow in epidemiology ${ }^{10}$, Bengt Haglund associate professor and senior researcher $^{1}$

${ }^{1}$ Centre for Pharmacoepidemiology, Department of Medicine Solna, Karolinska Institutet, Karolinska University Hospital, T2, SE-171 76 Stockholm, Sweden; ${ }^{2} \mathrm{THL}$ National Institute for Health and Welfare, Helsinki, Finland; ${ }^{3}$ Department of Pharmacoepidemiology, Norwegian Institute of Public Health, Oslo, Norway; ${ }^{4}$ Department of Public Health and Primary Health Care, University of Bergen, Norway; ${ }^{5}$ National Board of Health and Welfare, Stockholm, Sweden; ${ }^{6}$ Department of Pharmacy, University of Troms $\varnothing$, Norway; ${ }^{7}$ Nordic School of Public Health, Gothenburg, Sweden; ${ }^{8}$ Department of Clinical Epidemiology, Institute of Clinical Medicine, Aarhus University Hospital, Denmark; 'Department of Women's and Children's Health, Karolinska University Hospital and Institutet, Stockholm, Sweden; ${ }^{10}$ Centre of Public Health Sciences, School of Health Sciences, University of Iceland, Reykjavik, Iceland

\begin{abstract}
Objective To assess whether maternal use of selective serotonin reuptake inhibitors (SSRIs) increases the risk of persistent pulmonary hypertension in the newborn, and whether such an effect might differ between specific SSRIs.

Design Population based cohort study using data from the national health registers.
\end{abstract}

Setting Denmark, Finland, Iceland, Norway, and Sweden, 1996-2007. Participants More than 1.6 million infants born after gestational week 33.

Main outcome measures Risks of persistent pulmonary hypertension of the newborn associated with early and late exposure to SSRIs during pregnancy and adjusted for important maternal and pregnancy characteristics. Comparisons were made between infants exposed and not exposed to SSRIs.

Results Around 30000 women had used SSRIs during pregnancy and 11014 had been dispensed an SSRI later than gestational week 20 . Exposure to SSRIs in late pregnancy was associated with an increased risk of persistent pulmonary hypertension in the newborn: 33 of 11014 exposed infants (absolute risk 3 per 1000 liveborn infants compared with the background incidence of 1.2 per 1000); adjusted odds ratio 2.1 (95\% confidence interval 1.5 to 3.0). The increased risks of persistent pulmonary hypertension in the newborn for each of the specific SSRIs (sertraline, citalopram, paroxetine, and fluoxetine) were of similar magnitude. Filling a prescription with SSRIs before gestational week 8 yielded slightly increased risks: adjusted odds ratio 1.4 (95\% confidence interval 1.0 to 2.0 ).

Conclusions The risk of persistent pulmonary hypertension of the newborn is low, but use of SSRIs in late pregnancy increases that risk more than twofold. The increased risk seems to be a class effect.

\section{Introduction}

Persistent pulmonary hypertension of the newborn is a life threatening condition that occurs in up to 2 per 1000 liveborn infants and most often in those born full term or post term..$^{1-3}$ In this condition the pulmonary vascular resistance fails to decrease after birth and the ductus arteriosus must remain open to ensure circulation. Persistent pulmonary hypertension of the newborn and persistent fetal circulation are often used synonymously. 
Several perinatal risk factors for persistent pulmonary hypertension of the newborn are recognised, including maternal overweight, smoking, diabetes, or use of non-steroidal anti-inflammatory drugs during pregnancy. ${ }^{4}$ The pathophysiology is not clearly mapped out, but persistent pulmonary hypertension of the newborn might result from constricted pulmonary vasculature or because the vasculature is hypoplastic or remodelled. ${ }^{3}$ The constricted form is most commonly caused by meconium aspiration, the hypoplastic form can be seen in connection with diaphragmatic hernia, and presumably certain drugs can cause a remodelling of the vasculature.

Depression during pregnancy is common, and estimated rates vary from $7 \%$ to $25 \% .{ }^{5}$ Use of selective serotonin reuptake inhibitors (SSRIs) is increasing among pregnant women, and use in late pregnancy may be a risk factor for persistent pulmonary hypertension of the newborn. ${ }^{7}$ During the past 15 years six studies have specifically assessed this association, with inconsistent findings from no association to a sixfold increased risk. ${ }^{7-12}$ All the previous studies lacked statistical power for more detailed analyses, particularly the assessment of specific SSRIs.

To assess the association between SSRIs and persistent pulmonary hypertension of the newborn we carried out a multinational cohort study totalling more than 1.6 million births. Based on the findings in the previous studies we focused on exposure to SSRIs or specific SSRIs in late pregnancy. To address confounding by indication we also assessed the consequences of being exposed only in early pregnancy and calculated the risks for women with a previous psychiatric history and no use of SSRIs during pregnancy. As the mechanism behind a possible association between SSRIs and persistent pulmonary hypertension of the newborn is unknown but might be generated through increased levels of serotonin or norepinephrine, we also assessed the risks associated with other antidepressants with an effect on serotonin or norepinephrine activity. Finally, we assessed the risks after excluding infants with meconium aspiration, which is the most common cause of persistent pulmonary hypertension of the newborn.

\section{Methods}

We included women and their infants born in Denmark, Finland, Iceland, Norway, or Sweden between 1996 and 2007. Each Nordic country has national registers, which include prospectively collected information on the health of all inhabitants. All registers include the civil registration number of each resident, a unique number assigned at birth or immigration. From all five countries we obtained data from the medical birth registers, the prescription registers, and the cause of death registers. From the patient registers in Denmark, Iceland, Sweden, and Finland and from the Danish Psychiatric Central Register we included data on the mother's previous psychiatric diseases and infant diagnoses. Reporting to the registers is mandatory and regulated by national laws. The parliaments in the Nordic countries have on behalf of their populations given informed consent to be included in the registers. ${ }^{13}$

The Nordic birth registers include data on maternal characteristics, the pregnancy, delivery, and neonatal period. ${ }^{14}$ Complications are recorded according to the International Classification of Diseases and for the study period ICD-10 (10th revision) codes were used. Gestational age was primarily based on estimation of the last menstrual period using prenatal ultrasound. Since the mid-90s, ultrasound for determination of gestational length has been offered to all pregnant women in the Nordic countries. ${ }^{15-19}$ The prescription registers include data on dispensed items, substances, brand names, and formulations, together with the date of dispensing. All drugs are classified according to the World Health Organization Anatomical Therapeutic Chemical classification. In general, prescriptions are filled for a maximum of three months. ${ }^{20}$ The patient registers in Denmark, Finland, Iceland, and Sweden record information on admissions to hospital, including date of admission and discharge and primary and secondary diagnoses. The Danish Psychiatric Central Register includes information on psychiatric diseases. ${ }^{21}$ In each national cause of death register the date and cause of death are recorded for those who at the time of death were resident in the country. All diagnoses and causes of death are classified according to the current International Classification of Diseases.

\section{Participants}

We identified all singletons born after 231 gestational days (33 weeks) between 1996 and 2007. Only births were included from the years when prescription data were available. Accordingly we included births from: Denmark 1997-2007, Finland 1996-2006, Iceland 2003-7, Norway 2005-7, and Sweden 2006-7. From the registers we obtained information on persistent pulmonary hypertension of the newborn, level of delivery hospital, maternal smoking, body mass index in early pregnancy, year of birth, mode of delivery, gestational age at birth, birth weight, meconium aspiration, and maternal diseases recorded during pregnancy. Maternal diseases included epilepsy, malignancies, rheumatoid arthritis, juvenile arthritis, and arthritis in connection with psoriasis or inflammatory bowel disease, inflammatory bowel disease, systemic lupus erythematosus, and hypertension or pre-eclampsia. We also obtained information on the mothers' admissions to hospital for a psychiatric diagnosis during the 10 years before giving birth and identified women who had filled prescriptions for antidepressants, antidiabetes drugs, or non-steroidal anti-inflammatory drugs from three months before the start of pregnancy until delivery. Start of pregnancy was defined as the first day of the estimated last menstrual period according to the ultrasound examination in the first or second trimester. Small for gestational age was defined as a birth weight of two standard deviations or more below the mean birth weight according to sex specific fetal growth curves. ${ }^{22}$

\section{Exposures}

The SSRIs used during the study period and included in the analyses were fluoxetine, citalopram, paroxetine, sertraline, fluvoxamine, and escitalopram. In subanalyses we studied whether other antidepressants with an effect on serotonin activity or norepinephrine activity (clomipramine, venlafaxine, imipramine, amitriptyline, duloxetine, dosulepine, milnacipran, trazodone, nefazodone, and moklobemide) would affect the risks of persistent pulmonary hypertension of the newborn. We categorised use as ever use (from three months before the start of pregnancy until birth), as a filled prescription in late pregnancy (from 140 days after the start of pregnancy until birth), or in early pregnancy only (from three months before the start of pregnancy until a pregnancy length of 55 days).

\section{Outcome measures}

As a main outcome we included infants with persistent pulmonary hypertension of the newborn recorded as an ICD-10 code P29.3 or I27.0, if diagnosed within seven days of birth. In 
the countries where it was available we also evaluated infants with a diagnosis according to a national ICD-10 subcode of persistent pulmonary hypertension of the newborn (P29.3A in Denmark, P29.31 in Finland, and P29.3B in Sweden). Potential confounders included maternal smoking, age, body mass index, purchased non-steroidal anti-inflammatory drugs, and antidiabetes drugs, and diseases recorded during pregnancy, level of delivery hospital (university or non-university hospital), and infants' country of birth, birth year, and birth order.

\section{Statistical analyses}

We used logistic regression analysis to compare the risk of persistent pulmonary hypertension of the newborn with regard to exposure status of antidepressants during pregnancy, and computed crude and adjusted odds ratios with $95 \%$ confidence intervals. In our primary analyses we assessed risks after exposures to any SSRI and to specific SSRIs in late pregnancy. The analyses were repeated after excluding infants who also had a diagnosis of meconium aspiration and for exposure to the other antidepressants with effect on serotonin activity or norepinephrine activity. As smoking and body mass index in an analysis carried out in a subset did not confound the results we precluded these variables from further analyses. In additional analyses we assessed risks after exposures in early pregnancy. To test whether growth restriction or mode of delivery modified the effect of exposure to SSRIs, we carried out interaction analyses including small for gestational age and caesarean delivery as effect modifiers and tested how they affected the risk estimates by introducing them one by one into the model. We calculated the absolute risk for persistent pulmonary hypertension of the newborn after exposure to any SSRI in late pregnancy. Finally, we assessed the odds ratios of persistent pulmonary hypertension of the newborn for women with a previous hospital stay for a psychiatric disorder but not using antidepressants during pregnancy and for women with a previous psychiatric hospital stay and using antidepressants in late pregnancy.

All analyses were done using SAS software, version 9.2.

\section{Results}

In total, 1618255 singleton births were included in the study. Of these, $11014(0.7 \%)$ of the mothers had filled a prescription for an SSRI during late pregnancy and $17053(1.1 \%)$ in early pregnancy only. The mothers who had filled a prescription for an SSRI were generally older and more often smokers than mothers not using SSRIs. The exposed infants had a shorter gestational age at birth and were more often classified as small for gestational age (table $1 \Downarrow$ ).

Table $2 \Downarrow$ presents the distribution of the different SSRIs according to time of use. In late pregnancy, 627 women had filled a prescription for an antidepressant with an effect on serotonin activity or norepinephrine activity. The corresponding figure for early pregnancy only was 2503 . Of 63615 women with a previous psychiatric diagnosis, 54184 had not filled a prescription for any of the antidepressants during pregnancy. Among the 11014 infants exposed to an SSRI in late pregnancy, $33 \mathrm{had}$ a diagnosis of persistent pulmonary hypertension of the newborn, of whom three had meconium aspiration. The corresponding figure for those exposed to antidepressants with an effect on serotonin activity or norepinephrine activity was three. Of the 17053 infants exposed only in early pregnancy, 32 had persistent pulmonary hypertension of the newborn. The corresponding figure for the 158840 never exposed was 1935 . Tables $3 \Downarrow$ and $4 \Downarrow$ show the exposure by substance and time in pregnancy. Of the infants with persistent pulmonary hypertension of the newborn, three of the 33 infants exposed in late pregnancy died as well as 183 of the 1935 who were never exposed. Among the women with a previous admission to hospital for a psychiatric disorder, 114 infants had a diagnosis of persistent pulmonary hypertension of the newborn.

\section{Risks of exposure in late pregnancy}

The risk of persistent pulmonary hypertension of the newborn after exposure to any SSRI in late pregnancy was more than doubled: adjusted odds ratio 2.1 (95\% confidence interval 1.5 to 3.0, table 3). In the additional analyses assessing only infants with a diagnosis according to national subcodes for persistent pulmonary hypertension of the newborn, the increase in risk was 2.2 (1.2 to 3.9). After excluding infants with meconium aspiration, the risk estimates increased slightly. For fluoxetine, citalopram, sertraline, and paroxetine the risk estimates ranged from two to three (table 3). The risk estimate for escitalopram was lower, but imprecise. No infants with persistent pulmonary hypertension of the newborn had been exposed to fluvoxamine. Exposure to the other antidepressants with an effect on serotonin activity or norepinephrine activity also generated increased risks $(2.9,0.9$ to 8.9$)$. Neither small for gestational age nor caesarean delivery modified the risks ( $P>0.18)$, and the effects were not mediated through these variables. The absolute risk for persistent pulmonary hypertension of the newborn was 3 per 1000 .

\section{Risks of exposure in early pregnancy and after a previous psychiatric diagnosis}

Risks for persistent pulmonary hypertension of the newborn were slightly increased in association with exposure to SSRIs in early pregnancy, only (table 4). Exposure to the other antidepressants with effect on serotonin activity or norepinephrine activity did not increase the risk of persistent pulmonary hypertension of the newborn $(0.6,0.1$ to 2.3$)$. The odds ratios associated with exposure to specific SSRIs in early pregnancy varied from 0.3 to 1.9 .

For women with a previous admission to hospital for a psychiatric disorder the risk for persistent pulmonary hypertension of the newborn was slightly increased (1.3, 1.0 to 1.6) as well as after exclusion of infants with meconium aspiration $(1.3,1.1$ to 1.7$)$. The risk was not increased when estimated by the national subcodes $(1.0,0.6$ to 1.6$)$. The combination of a previous admission to hospital for a psychiatric disorder and exposure to an SSRI in late pregnancy yielded an adjusted odds ratio of 3.1 (1.9 to 4.9).

\section{Discussion}

In this large multinational cohort study we found a robust association between exposure to SSRIs in late pregnancy and persistent pulmonary hypertension of the newborn. Thus our findings constitute an important manifestation of the previous reported indications. ${ }^{710}$ In addition, the increased risks seemed to be a class effect as the risk estimates for the different SSRIs were of similar magnitude.

\section{Strengths and limitations of the study}

To ensure statistical power we included more than 1.6 million births, which allowed us to study specific SSRIs. Still, we identified only 33 infants exposed to an SSRI in late pregnancy and with a diagnosis of persistent pulmonary hypertension of the newborn. We used information from the Nordic health registers, and as the information is prospectively collected, recall 
bias can be precluded. To our knowledge the ICD code for our outcome of interest has not been validated in any of the Nordic countries; however, measurement of exposure and outcome variables in the registers is generally accurate and other diagnoses have been found to have high validity. ${ }^{23-25}$

Additional strengths of the study include its multinational and population based design, encompassing virtually all births in the Nordic countries, which should assure the generalisability of the study results. Potential limitations include information on drug intake during pregnancy. Exposure to antidepressants was measured as dispensed drugs, which does not necessarily imply use of a drug. ${ }^{26}$ However, fairly good agreement has been reported between use of antidepressants during pregnancy and dispensed drugs. ${ }^{27}{ }^{28}$ Also, misclassification of exposure would most likely be non-differential according to outcome status and, if anything, bias the risk estimates towards the null. We did not assess possible exposures to more than one antidepressant, but considering the short exposure periods of less than five months it seems unlikely to have occurred, at least to a greater extent. For the outcomes, we used the ICD codes recorded in the registers, and persistent pulmonary hypertension of the newborn shares the ICD code with symptomatic patent ductus arteriosus. However, as symptomatic patent ductus arteriosus is seen almost exclusively in very preterm infants, we assume that our restriction to include only infants born in gestational week 33 or later supports an association with persistent pulmonary hypertension of the newborn and not with symptomatic patent ductus arteriosus. This assumption is strengthened by our similar risk estimates, whether we used the broader ICD codes or the national subcodes. Also, the incidence of 1.2 per 1000 liveborn infants with persistent pulmonary hypertension of the newborn is in accordance with the expected number of affected infants. To further improve the identification of infants with the type of persistent pulmonary hypertension of the newborn, which might be associated with drug exposures, we excluded infants with meconium aspiration and found no major differences in risks.

\section{Indicating a serotonin effect}

The mechanism by which SSRIs may affect the pulmonary vasculature and cause persistent pulmonary hypertension of the newborn remains unknown. However, possible contributing factors include the accumulation of SSRIs in the lungs, together with the potential of serotonins to induce vasoconstriction and to mediate pulmonary arterial smooth muscle cell proliferation through the serotonin transporter. ${ }^{29-34}$ The increased risks associated with exposure to SSRI and to other antidepressants with selectivity for serotonin or norepinephrine in late pregnancy could support a causal effect of serotonin. Also, the similar increases in risk after use of specific types of SSRIs points to a common factor among the different substances.

\section{Comparison with other studies}

Our result of an increased risk of persistent pulmonary hypertension of the newborn in association with exposure to SSRI in late pregnancy is in accordance with what has been reported in two previous studies, except for our lower risk estimate. ${ }^{710}$ One of the studies ${ }^{10}$ also used register data, but only from the Swedish medical birth register, and exposure to SSRI was based on self reported use, mainly in early pregnancy. Accordingly, use in late pregnancy could not be satisfactorily assessed in that study. In contrast to the results reported by another study ${ }^{7}$ but in accordance with those by the Swedish register based study, ${ }^{10}$ we found slightly increased risks after exposure to SSRIs in early pregnancy. The estimates were, however, imprecise. Unexpectedly, we also found a slightly increased risk among women with a previous admission to hospital for a psychiatric disorder who had not been dispensed antidepressants from three months before pregnancy until birth. Some women might have been prescribed SSRIs before pregnancy or during early pregnancy but reduced their doses of SSRIs or stopped taking the drugs on planning or becoming aware of their pregnancy. Such behaviour could enable continued or resumed use of drugs later in pregnancy without them being newly dispensed. When the subcodes for persistent pulmonary hypertension of the newborn were used there was, however, no increased risk with a previous psychiatric disease, which strengthens our suspicion of an association with the drug and not the underlying disease. None the less, the findings of slightly increased risks in association with a previous psychiatric diagnosis or dispensing of an SSRI in early pregnancy merit further investigations.

\section{Conclusion}

Persistent pulmonary hypertension of the newborn is a rare disease and the absolute risks for this outcome for infants to mothers using SSRIs was as low as three infants per 1000 exposed. However, as the risk in association with treatment in late pregnancy seems to be more than doubled, we recommend caution when treating pregnant women with SSRIs. It is essential to plan the treatment and to weigh the risks of persistent pulmonary hypertension of the newborn when treating women in late pregnancy with those of relapse of depression and neonatal abstinence syndrome if therapy is interrupted. For women where treatment with an SSRI is the only or best option, the choice of substance seems to be of minor importance.

We thank Jørgen G Bramness, University of Oslo, Norway for his contributions to the design of the study.

Contributors: HK had full access to all of the data in the study, takes responsibility for the integrity of the data and the accuracy of the data analysis, and is guarantor. $\mathrm{HK}, \mathrm{KF}, \mathrm{MG}, \mathrm{MN}, \mathrm{UV}$, and $\mathrm{BH}$ conceived and designed the study. MA, AE, MN, RBN, UN, and $B H$ acquired the data in their countries. HK drafted the manuscript, obtained funding, and supervised the study. $\mathrm{BH}$ was responsible for the statistical analysis. All authors participated in interpreting the data and critically revising the manuscript.

Funding: This study was funded by the Swedish Pharmacy Company and by the authors' affiliations. The Swedish Pharmacy Company was not involved in the design and conduct of the study; collection, management, analysis, or interpretation of the data; and preparation, review, or approval of the manuscript.

Competing interests: All authors have completed the ICMJE uniform disclosure form at www.icmje.org/coi_disclosure.pdf (available on request from the corresponding author) and declare: no support from any organisation for the submitted work; no financial relationships with any organisations that might have an interest in the submitted work in the previous three years, no other relationships or activities that could appear to have influenced the submitted work.

Ethical approval: This study was approved by the regional ethical review board at the Karolinska Institutet, Sweden (No 2008/1371-31/4); the Danish Data Protection Agency and the National Board of Health, Denmark; the National Institute for Health and Welfare (THL), the Social Insurance Institution of Finland and Statistics Finland, Finland; the National Bioethics Committee and the Data Protection Authority in Iceland, Iceland; and the Norwegian Data Inspectorate, Norway.

Data sharing: Codes from the International Classification of Diseases and Anatomical Therapeutic Chemical for the listed diseases and drugs are available from the corresponding author at helle.kieler@ki.se. 


\section{What is already known on this topic}

The population incidence of persistent pulmonary hypertension of the newborn is 1.2 per 1000

It is unclear whether use of selective serotonin reuptake inhibitors (SSRIs) in pregnancy increases the risks of persistent pulmonary hypertension of the newborn

The use of SSRIs during pregnancy is increasing

Around $15 \%$ of infants with persistent pulmonary hypertension of the newborn will die

\section{What this study adds}

Use of SSRIs after gestational week 20 is associated with a doubled risk of persistent pulmonary hypertension of the newborn, to 3 per 1000 liveborn infants

Specific SSRIs have similar increased risks of persistent pulmonary hypertension of the newborn, which suggests a class effect Rare drug exposures and outcomes can be evaluated by combining data from the Nordic health registers

1 Farrow KN, Fliman P, Steinhorn RH. The diseases treated with ECMO: focus on PPHN. Semin Perinatol 2005;29:8-14

2 Perreault T. Persistent pulmonary hypertension of the newborn. Paediatr Respir Rev 2006; 7:S175-6.

3 Steinhorn RH. Neonatal pulmonary hypertension. Pediatr Crit Care Med 2010;11:S79-84. Hernandez-Diaz S, Van Marter LJ, Werler MM, Louik C, Mitchell AA. Risk factors for persistent pulmonary hypertension of the newborn. Pediatrics 2007;120:e272-82.

5 Bakker MK, Kolling P, van den Berg PB, de Walle HE, de Jong van den Berg LT. Increase in use of selective serotonin reuptake inhibitors in pregnancy during the last decade, a population-based cohort study from the Netherlands. Br J Clin Pharmacol 2008:65:600-6.

6 Cohen LS, Altshuler LL, Harlow BL, Nonacs R, Newport DJ, Viguera AC, et al. Relapse of major depression during pregnancy in women who maintain or discontinue antidepressant treatment. JAMA 2006;295:499-507.

7 Chambers CD, Hernandez-Diaz S, Van Marter LJ, Werler MM, Louik C, Jones KL, et al. Selective serotonin-reuptake inhibitors and risk of persistent pulmonary hypertension of the newborn. N Engl J Med 2006:354:579-87.

8 Andrade SE, McPhillips H, Loren D, Raebel MA, Lane K, Livingston J, et al. Antidepressant medication use and risk of persistent pulmonary hypertension of the newborn. Pharmacoepidemiol Drug Saf 2009;18:246-52.

9 Chambers CD, Johnson KA, Dick LM, Felix RJ, Jones KL. Birth outcomes in pregnant women taking fluoxetine. N Engl J Med 1996;335:1010-5.

10 Kallen B, Olausson PO. Maternal use of selective serotonin re-uptake inhibitors and persistent pulmonary hypertension of the newborn. Pharmacoepidemiol Drug Saf 2008;17:801-6.

11 Wichman CL, Moore KM, Lang TR, St Sauver JL, Heise RH Jr, Watson WJ. Congenital heart disease associated with selective serotonin reuptake inhibitor use during pregnancy. Mayo Clin Proc 2009;84:23-7.

12 Wilson KL, Zelig CM, Harvey JP, Cunningham BS, Dolinsky BM, Napolitano PG. Persisten pulmonary hypertension of the newborn is associated with mode of delivery and not with maternal use of selective serotonin reuptake inhibitors. Am J Perinatol 2011;28:19-24.

13 Rosen M. National health data registers: a Nordic heritage to public health. Scand J Public Health 2002;30:81-5.

14 Gissler M, Louhiala P, Hemminki E. Nordic medical birth registers in epidemiological research. Eur J Epidemiol 1997;13:169-75.

15 Backe B. [Routine ultrasonography in obstetric care in Norway, 1994]. Tidsskr Nor Laegeforen 1997;117:2314-5.

16 Geirsson RT. [Ultrasound screening at 18-20 weeks.]. Laeknabladid 2001;87:403-7.

17 Hogberg U, Larsson N. Early dating by ultrasound and perinatal outcome. A cohort study. Acta Obstet Gynecol Scand 1997;76:907-12.

18 Qvist I, Iversen M, Skajaa K. [Attitudes and expectations of pregnant women to routine ultrasonography in early pregnancy]. Ugeskr Laeger 2002;164:902-5.

19 Salonen R, Ammala P. Prenatal diagnosis in Finland. Eur J Hum Genet 1997;5:22-5.
20 Furu K, Wettermark B, Andersen M, Martikainen JE, Almarsdottir AB, Sorensen HT. The Nordic countries as a cohort for pharmacoepidemiological research. Basic Clin Pharmacol Toxicol 2010;106:86-94.

21 Munk-Jorgensen P, Mortensen PB. The Danish Psychiatric Central Register. Dan Med Bull 1997;44:82-4.

22 Marsal K, Persson PH, Larsen T, Lilja H, Selbing A, Sultan B. Intrauterine growth curves based on ultrasonically estimated foetal weights. Acta Paediatr 1996;85:843-8.

23 Cnattingius S, Ericson A, Gunnarskog J, Kallen B. A quality study of a medical birth registry. Scand J Soc Med 1990;18:143-8.

24 Gissler M, Teperi J, Hemminki E, Merilainen J. Data quality after restructuring a nationa medical registry. Scand J Soc Med 1995;23:75-80.

25 Nickelsen TN. [Data validity and coverage in the Danish National Health Registry. A literature review]. Ugeskr Laeger 2001;164:33-7.

26 Elliott R. Non-adherence to medicines: not solved but solvable. J Health Serv Res Policy 2009;14:58-61

27 Olesen C, Sondergaard C, Thrane N, Nielsen GL, de Jong-van den Berg L, Olsen J. Do pregnant women report use of dispensed medications? Epidemiology 2001;12:497-501.

28 Stephansson O, Granath F, Svensson T, Haglund B, Ekbom A, Kieler H. Drug use during pregnancy in Sweden—assessed by the Prescribed Drug Register and the Medical Birth Register. Clin Epidemiol 2011;3:43-50.

29 Eddahibi S, Raffestin B, Hamon M, Adnot S. Is the serotonin transporter involved in the pathogenesis of pulmonary hypertension? J Lab Clin Med 2002;139:194-201.

30 MacLean MR, Dempsie Y. Serotonin and pulmonary hypertension-from bench to bedside? Curr Opin Pharmacol 2009;9:281-6.

31 McMahon TJ, Hood JS, Nossaman BD, Kadowitz PJ. Analysis of responses to serotonin in the pulmonary vascular bed of the cat. J Appl Physiol 1993;75:93-102.

32 Runo JR, Loyd JE. Primary pulmonary hypertension. Lancet 2003;361:1533-44.

33 Suhara T, Sudo Y, Yoshida K, Okubo Y, Fukuda H, Obata T, et al. Lung as reservoir for antidepressants in pharmacokinetic drug interactions. Lancet 1998;351:332-5.

34 Yaron I, Shirazi I, Judovich R, Levartovsky D, Caspi D, Yaron M. Fluoxetine and amitriptyline inhibit nitric oxide, prostaglandin E2, and hyaluronic acid production in human synovial cells and synovial tissue cultures. Arthritis Rheum 1999;42:2561-8.

\section{Accepted: 31 October 2011}

\section{Cite this as: BMJ 2012:344:d8012}

This is an open-access article distributed under the terms of the Creative Commons Attribution Non-commercial License, which permits use, distribution, and reproduction in any medium, provided the original work is properly cited, the use is non commercial and is otherwise in compliance with the license. See: http://creativecommons.org/licenses/by$\mathrm{nc} / 2.0 /$ and http://creativecommons.org/licenses/by-nc/2.0/legalcode. 


\section{Tables}

\section{Table 1| Maternal and infant characteristics by exposure to selective serotonin reuptake inhibitors}

\section{Characteristics}

Maternal age (years)

\begin{tabular}{|c|c|c|c|}
\hline$\leq 24$ & $5473(18.2)$ & $258474(16.3)$ & $263947(16.3)$ \\
\hline $25-34$ & $18105(60.1)$ & $1053684(66.3)$ & $1071789(66.2)$ \\
\hline $35-44$ & $6472(21.5)$ & $274208(17.3)$ & $280680(17.3)$ \\
\hline$\geq 45$ & $65(0.2)$ & $1774(0.1)$ & $1839(0.1)$ \\
\hline \multicolumn{4}{|l|}{ Birth order: } \\
\hline 1 & $12930(42.9)$ & $669026(42.1)$ & $681956(42.1)$ \\
\hline 2 or 3 & $14101(46.8)$ & $798481(50.3)$ & $812582(50.2)$ \\
\hline$\geq 4$ & $2964(9.8)$ & $113758(7.2)$ & $116722(7.2)$ \\
\hline Missing & $120(0.4)$ & $6875(0.4)$ & $6995(0.4)$ \\
\hline \multicolumn{4}{|c|}{ Gestational age (weeks): } \\
\hline $33-36$ & $1629(5.4)$ & $60758(3.8)$ & 62387 (3.9) \\
\hline $37-41$ & 27065 (89.9) & $1425414(89.8)$ & $1452479(89.8)$ \\
\hline $42-45$ & $1421(4.7)$ & $101968(6.4)$ & $103389(6.4)$ \\
\hline \multicolumn{4}{|c|}{ Small for gestational age*: } \\
\hline No & $29164(96.8)$ & $1545233(97.3)$ & 1574397 (97.3) \\
\hline Yes & $891(3.0)$ & $38438(2.4)$ & $39329(2.4)$ \\
\hline Missing data & $60(0.2)$ & $4469(0.3)$ & $4529(0.3)$ \\
\hline \multicolumn{4}{|c|}{ Smoking in early pregnancy: } \\
\hline No & 18945 (62.9) & $1246072(78.5)$ & $1265017(78.2)$ \\
\hline Yes & $8303(27.6)$ & $244258(15.4)$ & $252561(15.6)$ \\
\hline Missing data & $2867(9.5)$ & $97810(6.2)$ & $100677(6.2)$ \\
\hline \multicolumn{4}{|l|}{ Mode of delivery: } \\
\hline Vaginal & 23768 (78.9) & $1334843(84.1)$ & $1358611(84.0)$ \\
\hline Caesarean section & $5721(19.0)$ & 216926 (13.7) & $222647(13.8)$ \\
\hline Missing data & $626(2.1)$ & $36371(2.3)$ & 36997 (2.3) \\
\hline \multicolumn{4}{|c|}{ Maternal use of NSAIDs: } \\
\hline No & $26318(87.4)$ & $1486008(93.6)$ & $1512326(93.5)$ \\
\hline Yes & $3797(12.6)$ & $102132(6.4)$ & $105929(6.5)$ \\
\hline \multicolumn{4}{|c|}{ Maternal use of drugs for diabetes: } \\
\hline No & $29512(98.0)$ & $1572534(99.0)$ & $1602046(99.0)$ \\
\hline Yes & $603(2.0)$ & $15606(1.0)$ & $16209(1.0)$ \\
\hline \multicolumn{4}{|c|}{ Apgar score at 5 minutes: } \\
\hline $0-6$ & $332(1.1)$ & $8752(0.6)$ & $9084(0.6)$ \\
\hline $7-10$ & $18382(61.0)$ & $973229(61.3)$ & $991611(61.3)$ \\
\hline Missing data & 11401 (37.9) & $606159(38.2)$ & $617560(38.2)$ \\
\hline
\end{tabular}

NSAIDs $=$ non-steroidal anti-inflammatory drugs

*Less than 2 standard deviations of sex specific mean birth weight. 
Table 2| Selective serotonin reuptake inhibitors (SSRIs) according to time of dispensing (length of gestation)

\begin{tabular}{lccc} 
& \multicolumn{3}{l}{ No of women dispensed SSRIs (per 1000) } \\
\cline { 2 - 4 } Drugs & <week 8 only & 2week 20 & Ever \\
Any SSRI & $17053(10.5)$ & $11014(6.8)$ & $30115(18.6)$ \\
\hline Fluoxetine & $3899(2.4)$ & $3315(2.0)$ & $7988(4.9)$ \\
\hline Citalopram & $6816(4.2)$ & $3294(2.0)$ & $10820(6.7)$ \\
\hline Paroxetine & $2293(1.4)$ & $1281(0.8)$ & $3798(2.3)$ \\
\hline Sertraline & $3396(2.1)$ & $2843(1.8)$ & $6696(4.1)$ \\
\hline Fluvoxamine & $278(0.2)$ & $112(0.1)$ & $405(0.3)$ \\
\hline Escitalopram & $2510(1.6)$ & $557(0.3)$ & $3247(2.0)$ \\
\hline
\end{tabular}


Table 3| Exposure to selective serotonin reuptake inhibitors (SSRIs) in gestational week 20 or later and risk for persistent pulmonary hypertension of the newborn

\begin{tabular}{|c|c|c|c|c|}
\hline \multirow[b]{2}{*}{ Drugs } & \multicolumn{2}{|c|}{$\begin{array}{c}\text { No of infants with persistent pulmonary hypertension of } \\
\text { the newborn (per 1000) }\end{array}$} & \multicolumn{2}{|c|}{ Odds ratio $(95 \% \mathrm{Cl})$} \\
\hline & Not exposed & Exposed & Unadjusted & Adjusted* \\
\hline Any SSRI & $1899(1.2)$ & $33(3.0)$ & 2.5 (1.8 to 3.6$)$ & $2.1(1.5$ to 3.0$)$ \\
\hline Fluoxetine & $1952(1.2)$ & $9(2.7)$ & 2.3 (1.2 to 4.3$)$ & $2.0(1.0$ to 3.8$)$ \\
\hline Citalopram & $1936(1.2)$ & $11(3.3)$ & $2.8(1.5$ to 5.0$)$ & $2.3(1.2$ to 4.1$)$ \\
\hline Paroxetine & $1959(1.2)$ & $5(3.9)$ & $3.2(1.3$ to 7.8$)$ & $2.8(1.2$ to 6.7$)$ \\
\hline Sertraline & $1949(1.2)$ & $10(3.5)$ & $2.9(1.6$ to 5.4$)$ & 2.3 (1.3 to 4.4$)$ \\
\hline Escitalopram & $1966(1.2)$ & $1(1.8)$ & 1.5 (0.2 to 10.5$)$ & $1.3(0.2$ to 9.5$)$ \\
\hline
\end{tabular}

${ }^{*}$ Adjusted for maternal age, dispensed non-steroidal anti-inflammatory drugs and antidiabetes drugs, pre-eclampsia, chronic diseases during pregnancy, country of birth, birth year, level of delivery hospital, and birth order. 
Table 4| Exposure to selective serotonin reuptake inhibitors (SSRIs) before gestational week 8 and risk for persistent pulmonary hypertension of the newborn

\begin{tabular}{lcccc} 
& \multicolumn{2}{c}{$\begin{array}{c}\text { No of infants with persistent pulmonary hypertension of the } \\
\text { newborn (per 1000) }\end{array}$} & \multicolumn{2}{c}{ Odds ratio (95\% Cl) } \\
\cline { 2 - 5 } Drugs & Not exposed & Exposed & Unadjusted & Adjusted \\
Any SSRI & $1899(1.2)$ & $32(1.9)$ & $1.6(1.1$ to 2.2$)$ & $1.4(1.0$ to 2.0$)$ \\
\hline Fluoxetine & $1952(1.2)$ & $7(1.8)$ & $1.5(0.7$ to 3.1$)$ & $1.3(0.6$ to 2.8$)$ \\
\hline Citalopram & $1936(1.2)$ & $17(2.5)$ & $2.1(1.3$ to 3.4$)$ & $1.8(1.1$ to 3.0$)$ \\
\hline Paroxetine & $1959(1.2)$ & $4(1.7)$ & $1.4(0.5$ to 3.8$)$ & $1.3(0.5$ to 3.5$)$ \\
\hline Sertraline & $1949(1.2)$ & $9(2.7)$ & $2.2(1.1$ to 4.2$)$ & $1.9(1.0$ to 3.6$)$ \\
\hline Escitalopram & $1966(1.2)$ & $1(0.4)$ & $0.3(0.0$ to 2.3$)$ & $0.3(0.0$ to 2.2$)$ \\
\hline
\end{tabular}

${ }^{*}$ Adjusted for maternal age, dispensed non-steroidal anti-inflammatory drugs and antidiabetes drugs, pre-eclampsia, chronic diseases during pregnancy, country of birth, birth year, level of delivery hospital, and birth order. 